\title{
An Efficient Implementation of Context-aware Saliency Using a Stochastic Approach
}

\author{
Geurim Yun ${ }^{1}$, Heekyung Yang ${ }^{2}$ and Kyungha Min ${ }^{1}$ \\ ${ }^{1}$ Dept. of Digital Media, Sangmyung Univ. \\ ${ }^{2}$ Dept. of Computer Science, Graduate School, Sangmyung UNiv. \\ minkh@smu.ac.kr
}

\begin{abstract}
The computation of saliency from an image and a video is an interesting challenge in image processing and computer vision. Context-aware saliency, which addresses the saliency based on the geometric structure of an image, is known as one of the most powerful schemes for computing saliency. An obstacle of the context-aware scheme is the heavy computation load. We reduce computational loads in a great scale by applying the dart throwing algorithm.
\end{abstract}

Keywords: saliency; context-aware; stochastic; dart throwing

\section{Introduction}

Saliency is one of the most challenging properties of visual contents. Many schemes proposed for detecting saliency can be classified into three categories: those schemes using local properties $[5,6,8,10,3,4]$, those using global properties $[2,16,11]$ and those using both properties $[9,15,14,17]$. Among them, context-aware scheme [14] is recognized to present most outstanding result in detecting saliency. This scheme, however, suffers from heavy computational loads that prohibits from real-time saliency detection.

To address this problem, we apply a blue noise generation scheme for reducing the computational load. We sample $k$ important pixels from $n \times n$ pixels of an image and estimate the saliency at the sampled points. For the sampling, we build a strategy that evaluates the importance of the pixels and assigns the order of sampling. Our strategy is constructed based on the dart throwing algorithm [1]. Using this strategy, the noise of higher gradient has smaller radius than those of lower gradient. This property allows the blue noise distribution of the positions where the saliency is estimated. In our experiment, the computation time required for the saliency detection is reduced in 10 20\% of the time required for the original context-aware saliency detection [14] while our scheme produces almost similar results.

The overview of our algorithm is illustrated in Figure 1. In the first step, we sample $k$ locations where the saliency detection is estimated using the dart throwing algorithm. In the second, we estimate the saliency at the sampled locations. Finally, we propagate the estimated saliencies to the neighboring positions and build a saliency map. 


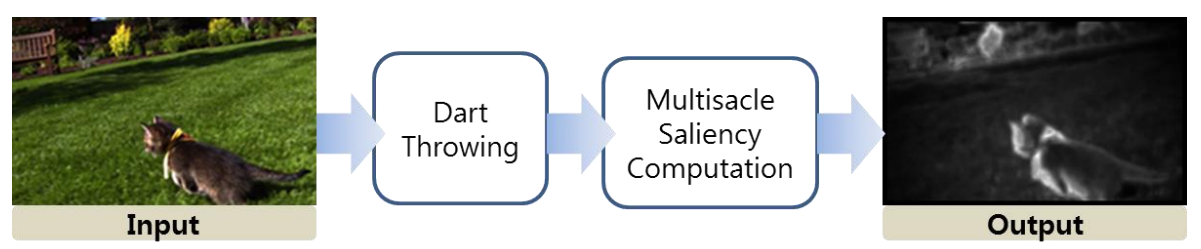

Figure 1. The overview of the algorithm

\section{Related work}

Most of the local schemes estimate saliency using low-level features and a bottom-up model. Itti et al., [5] have presented a saliency estimation scheme based on neuronal architecture of the visual system. Later, Itti and Koch [6] extended their previous work by analyzing five important trends in computing focal visual attention. Bruce and Tsotsos [8] proposed bottom-up overt attention method based on Shannon's selfinformation measure using a neural circuit. Meur et al., [10] developed a coherent model for bottom-up visual attention using the current understanding of human visual system behavior including contrast sensitivity functions, perceptual decomposition, visual masking and center-surround interactions. Walther and Koch [3] proposed a biologically plausible model of forming and attending to proto-objects, which is defined as volatile units of visual information accessed by selective attention and subsequently validated as actual objects. Harel et al., [4] presented a graph-based visual saliency model, which is a novel bottom-up visual saliency model. Their model forms activation maps on certain feature channels and normalize them by highlighting conspicuity and by admitting combination with other maps.

Some schemes exploit global feature analysis algorithms such as Fourier transform to detect saliency. Hou and Zhang [16] presented a saliency detection model, which is independent of features, categories or other forms of prior knowledge of the objects. Guo et al., [2] proposed the phase spectrum of the Fourier transform saliency detection method to obtain the location of salient areas. Achanta et al., [11] introduced a method for salient region detection with full resolution saliency map and well-defined boundaries of salient objects. Their model exploits features of color and luminance.

Some schemes combine both local and global approaches. Boiman and Irani [9] proposed a scheme that detects irregularities in visual data by composing a new observed image region or a new video segment using chunks of data extracted from previous visual examples. Liu et al., [15] presented a scheme that detects a salient object in an input image as an image segmentation problem by separating the salient object from the image background. Seo and Milanfar [17] developed a bottom-up unified framework for static and space-time saliency detection. Visual saliency is computed using self-resemblance measure. The resulting saliency map indicates that its pixel values represent the statistical likelihood of saliency of a feature matrix. Goferman et al., [14] presented a context-aware scheme for detecting saliency by considering a multi-scale approach. The saliency map is utilized in resizing and compressing a photograph while preserving its contents $[7,12]$ or composing a collage image [13]. 


\section{Sampling}

We sample $k$ pixels among $n \times n$ pixels on the image. Our strategy to sample the pixels is to control the sampling possibility according to the magnitude of gradient at the pixel. The outline of our strategy is summarized in Figure 2 and the result of the noise is in Figure 3. The keypoint is to sample a position from the image and test whether the sampled position is acceptable or not. We accept the sampled position if it lies outside the radii of the generated points. After the accepting the position, we assign a radius for the sampled position according to the gradient estimated at the position by $r_{i}=1 /\left(g_{i}+0.1\right)$, where $r_{i}$ is the radius and $g_{i}$ is the gradient. According to the dart throwing algorithm, we reduce the radii of the sampled positions as the sampling proceeds.

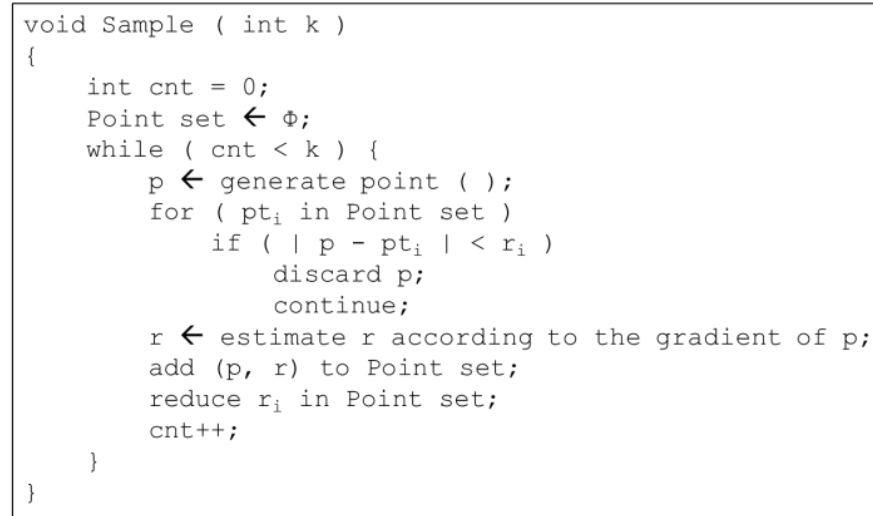

Figure 2. The outline of the sampling algorithm

\section{Saliency Detection}

The context-aware saliency detection scheme proposed by Goferman et al., [14] estimates the saliency at each pixel using a multi-scale approach. At each scale, we compute the difference of color and position between the sampled $k$ pixels and all the pixels in the image.

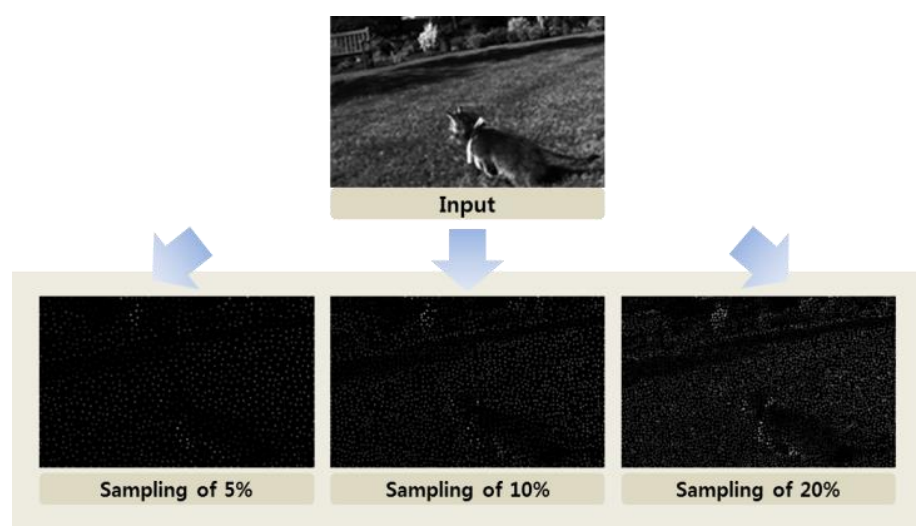

Figure 3. Sampling results of $5 \%, 10 \%$ and $20 \%$. 
At a pixel, we assign $m$, the size of a patch and build a vector of $m \times m$ neighbor pixels of the sampled pixel. $d_{c}\left(p_{i}, p_{j}\right)$, the Euclidean distance of the color of the patches of the pixels $p_{i}$ and ${ }_{\mathrm{pj}}$ is estimated as follows:

$$
d_{c}\left(p_{i}, p_{j}\right)=\sum_{l=1}^{m \times m}\left|C^{i}{ }_{l}-C^{j}{ }_{l}\right|
$$

Note that $C_{l}^{i}$ and $C_{l}^{j}$ are the color of $l$-th pixels of the patch from $\mathrm{p}_{\mathrm{i}}$ and $\mathrm{p}_{\mathrm{j}} . \mathrm{d}_{\mathrm{p}}\left(\mathrm{p}_{\mathrm{i}}, \mathrm{p}_{\mathrm{j}}\right)$ denotes the distance between the pixels $p_{i}$ and $p_{j}$. The final difference between pixels $p_{i}$ and $p_{j}$ is estimated as follows:

$$
d\left(p_{i}, p_{j}\right)=\frac{d_{c}\left(p_{i}, p_{j}\right)}{1+c \cdot d_{p}\left(p_{i}, p_{j}\right)}
$$

$S_{i}^{m}$, the saliency of a pixel $p_{i}$ with scale $m$ is estimated as follows:

$$
S_{i}{ }^{m}=1-\exp \left(\frac{-1}{K} \sum_{k=1}^{K} d\left(p_{i}, p_{j}\right)\right)
$$

Note that the $p_{k}$ are the pixels whose $\mathrm{d}\left(p_{i}, p_{j}\right)$ value is highest among all the pixels. The final saliency $S_{i}$ is estimated by averaging the saliencies of various scales as follows:

$$
S_{i}=\frac{1}{M} \sum_{a=1}^{m} S_{i}^{a}
$$

The process of saliency detection is illustrated in Figure 4.

\section{Implementation and Results}

We have implemented our algorithm in a personal computer with Pentium i7 CPU and 4 GB main memory. In our implementation we have sampled $10 \%$ of pixels from the input image and computed saliency. We reduce the comparison by $10 \%$ and improve the computational loads in a great scale. For three input images in Figure 5, we compare the computation time of our algorithm with [14] in Figure 6. We show that our scheme reduces the computation time of [14] to $10 \%$ scale.

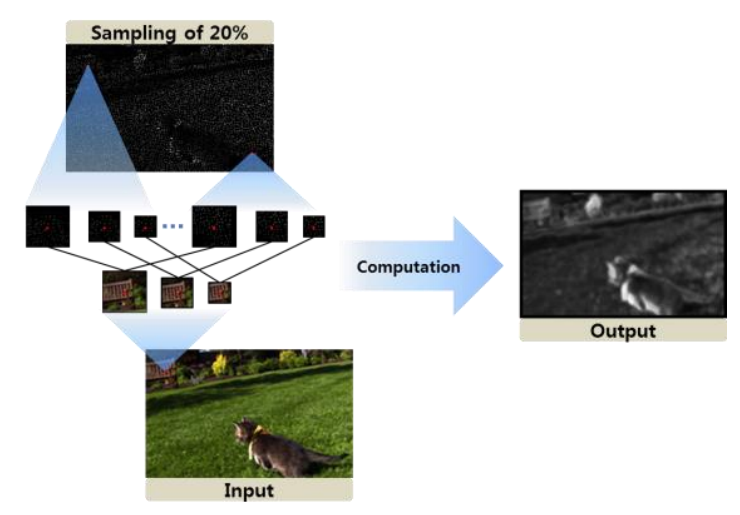

Figure 4. The process of saliency detection 


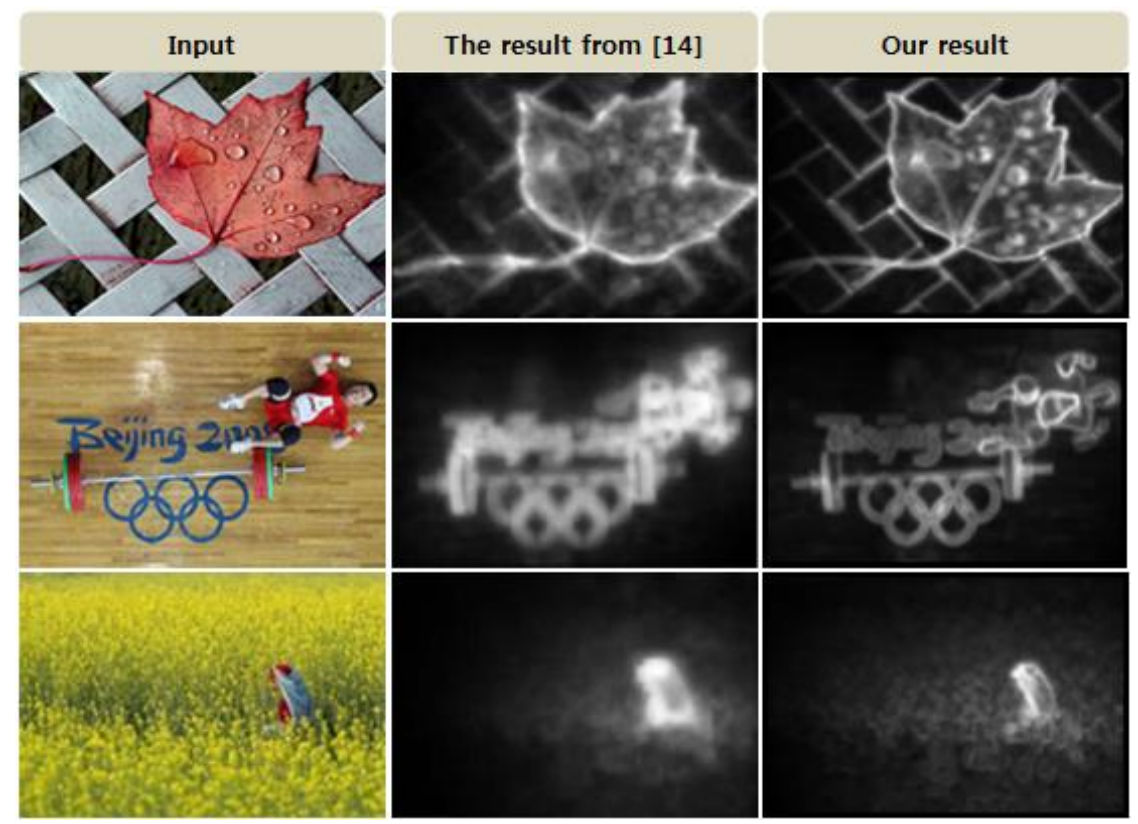

Figure 5. The result of our work and comparison with those from [14]

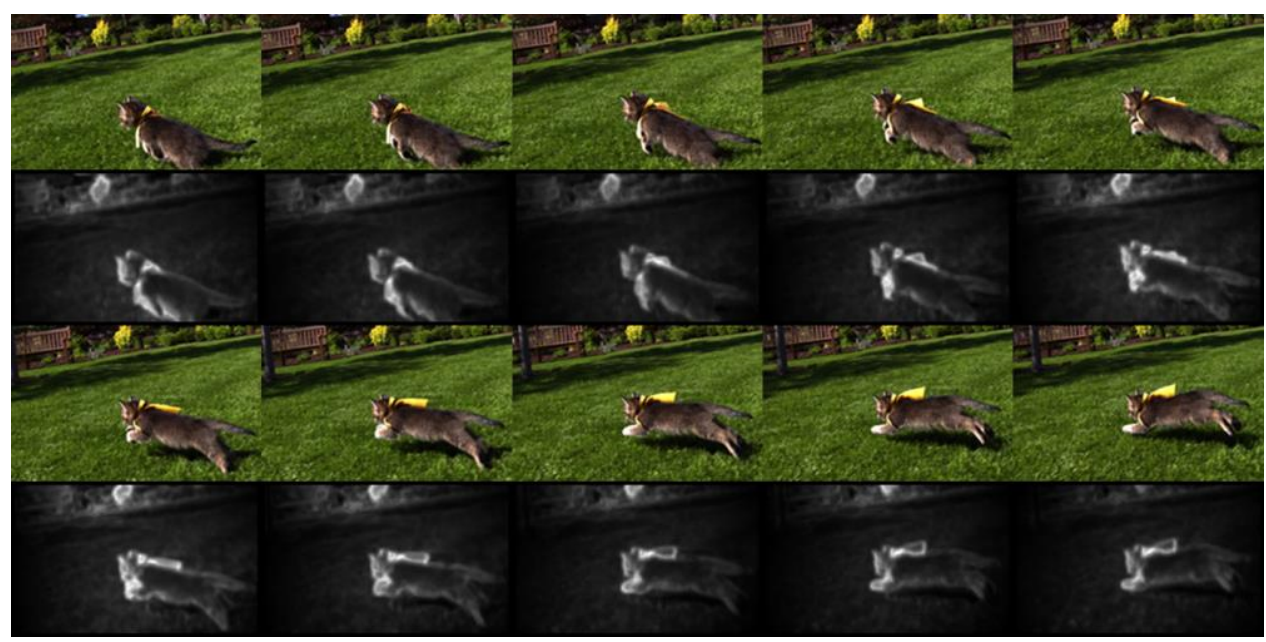

Figure 6. Tracking of objects using saliency

\section{Conclusion and Future Work}

In this paper, we have presented an improved saliency detection algorithm with a stochastic approach. We used a dart throwing algorithm to sample the important pixels from an image and reduced the computation time in $10 \%$ with similar saliency detection results. We further improve our algorithm to develop a real-time saliency-based object tracking in a video.

\section{Acknowledgements}

This research was supported by a 2013 Research Grant from Sangmyung University. 


\section{References}

[1] M. McCool and E. Fiume, "Hierarchical poisson disk sampling distributions", Proc. Graphics interface, (1992), pp. 94-105.

[2] Guo, Q. Ma and L. Zhang, "Spatio-Temporal Saliency Detection Using Phase Spectrum of Quaternion Fourier Transform", Proc. IEEE Conf. Comp. Vis. and Patt. Recog., (2008), pp. 1-8.

[3] D. Walther and C. Koch, "Modeling Attention to Salient Proto-Objects", Neural Networks, vol. 19, no. 9, (2006), pp. 1395-1407.

[4] J. Harel, C. Koch and P. Perona, "Graph-Based Visual Saliency", Advances in Neural Information Processing Systems, vol. 19, (2007), pp. 545-552.

[5] L. Itti, C. Koch and E. Niebur, "A Model of Saliency-Based Visual Attention for Rapid Scene Analysis", IEEE Trans. Pattern Analysis and Machine Intelligence, vol. 20, no. 11, (1998) November, pp. 1254-1259.

[6] L. Itti and C. Koch, "Computational Modeling of Visual Attention”, Nature Rev. Neuroscience, vol. 2, no. 3, (2001), pp. 194-204.

[7] M. Rubinstein, A. Shamir and S. Avidan, "Improved Seam Carving for Video Retargeting", ACM Trans. Graph., vol. 27, no. 3, (2008), pp. 16.

[8] N. Bruce and J. Tsotsos, "Saliency Based on Information Maximization", Advances in Neural Information Processing Systems, vol. 18, (2006), pp. 155-162.

[9] O. Boiman and M. Irani, "Detecting Irregularities in Images and in Video", Int'1 J. Computer Vision, vol. 74, no. 1, (2007), pp. 17-31.

[10] O. Le Meur, P. Le Callet, D. Barba and D. Thoreau, "A Coherent Computational Approach to Model Bottomup Visual Attention”, IEEE Trans. Pattern Analysis and Machine Intelligence, vol. 28, no. 5, (2006) May, pp. 802-817.

[11] R. Achanta, S. Hemami, F. Estrada and S. Susstrunk, "Frequency-Tuned Salient Region Detection", Proc. IEEE Conf. Computer Vision and Pattern Recognition, (2009), pp. 1597-1604.

[12] S. Avidan and A. Shamir, "Seam Carving for Content-AwareImage Resizing", ACM Trans. Graphics, vol. 26, no. 3, (2007), pp. 10.

[13] S. Goferman, A. Tal and L. Zelnik-Manor, "Puzzle-Like Collage", Computer Graphics Forum, vol. 29, (2010), pp. 459-468.

[14] S. Goferman, L. Zelnik-Manor and A. Tal, "Context-Aware Saliency Detection”, Proc. IEEE Transactions on Pattern Analysis and Machine Intelligence, vol. 34, no. 10, (2012).

[15] T. Liu, J. Sun, N. Zheng, X. Tang and H. Shum, "Learning to Detect a Salient Object", Proc. IEEE Conf. Computer Vision and Pattern Recognition, (2007).

[16] X. Hou and L. Zhang, "Saliency Detection: A Spectral Residual Approach", Proc. IEEE Conf. Computer Vision and Pattern Recognition, (2007), pp. 1-8.

[17] H. Seo and P. Milanfar, "Static and Space-Time Visual Saliency Detection by Self-Resemblance", J. Vision, vol. 9, no. 12, (2009), pp. 1-27.

\section{Authors}

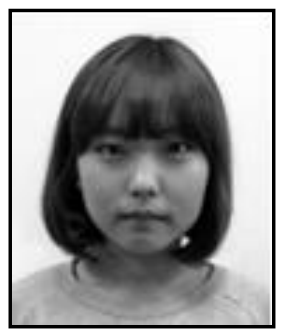

\section{Geurim Yun}

She is an undergraduate student at Dept. of Digital Media, Graduate School, Sangmyung Univ. Her research interest is computer graphics and image processing 


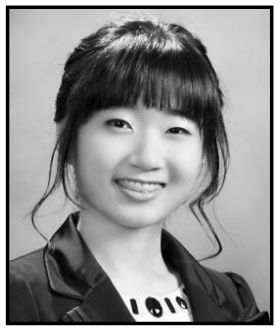

\section{Heekyung Yang}

Heekyung Yang is a Ph.D candidate at Dept. of Computer Science, Graduate School, Sangmyung Univ. She got her M.S. at Sangmyung Univ. at 2012. Her research interest is computer graphics and image processing.

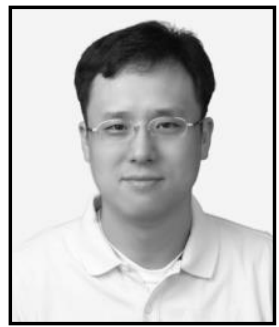

\section{Kyungha Min}

Kyungha Min got his Ph.D at POSTECH at 2000. His research interest is computer graphics and image processing. Currently he is at Dept. of Digital Media, Sangmyung Univ., Seoul, Korea. 
International Journal of Multimedia and Ubiquitous Engineering Vol.8, No.6 (2013) 